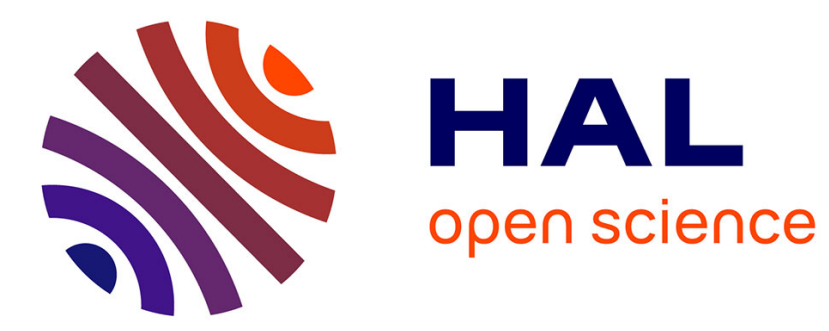

\title{
Conception avec règles en lambda d'une ROM 4-valuée
}

\author{
D. Etiemble, J. Ehrlich, B. Nateghi, E. Lavelle
}

\section{To cite this version:}

D. Etiemble, J. Ehrlich, B. Nateghi, E. Lavelle. Conception avec règles en lambda d'une ROM 4valuée. Revue de Physique Appliquée, 1985, 20 (2), pp.71-75. 10.1051/rphysap:0198500200207100 . jpa-00245308

\section{HAL Id: jpa-00245308 https://hal.science/jpa-00245308}

Submitted on 1 Jan 1985

HAL is a multi-disciplinary open access archive for the deposit and dissemination of scientific research documents, whether they are published or not. The documents may come from teaching and research institutions in France or abroad, or from public or private research centers.
L'archive ouverte pluridisciplinaire HAL, est destinée au dépôt et à la diffusion de documents scientifiques de niveau recherche, publiés ou non, émanant des établissements d'enseignement et de recherche français ou étrangers, des laboratoires publics ou privés. 


\title{
Conception avec règles en lambda d'une ROM 4-valuée
}

\author{
D. Etiemble $\left({ }^{*}\right)$, J. Ehrlich $\left({ }^{*}\right)$, B. Nateghi $\left({ }^{*}\right)$ et E. Lavelle $(* *)$ \\ (*) Université P. et M. Curie, Paris VI, 75230 Paris Cedex 05, France \\ (**) SAGEM, Pontoise, France
}

(Reçu le 16 mai 1984, accepté le 15 novembre 1984)

\begin{abstract}
Résumé. - L'utilisation de ROMS 4-valuées, avec 2 bits par cellule, est une solution intéressante pour diminuer la taille des mémoires à lecture seule $(\mathrm{ROM})$ de grosse capacité. Alors que la publication originale d'Intel ne donnait aucune information sur les tolérances technologiques, nous montrons la "faisabilité " d'une conception avec règles en $\lambda$ sur le CMP français (Circuit Multi-Projets) d'une ROM 4-valuée que nous comparons avec la ROM binaire de même capacité utilisant les mêmes règles de dessin.
\end{abstract}

\begin{abstract}
ROMS, with 2-bit per cell, are a valuable solution to increase memory density of Read Only Memories. Intel presentation of a 4-valued ROM didn't give details on the technological tolerances that are needed. We show that a 4-valued ROM can be designed with the French MultiProject Circuit (MPC) simplified design rules and large parameter tolerances. We compare it with the corresponding binary ROM using the same design rules.
\end{abstract}

\section{Introduction.}

En 1981, Intel a présenté une ROM 4-valuée NMOS utilisée dans les circuits 8087 et IAPX 432 [1]. En 1983, Motorola a annoncé une ROM 4-valuée CMOS [2]. Ceci correspond à un intérêt croissant pour toute solution permettant de diminuer la surface des mémoires mortes (ROM) de grande capacité, utilisée pour le stockage du microcode des circuits VLSI microprogrammés [3], les générateurs de caractères... Bien que la réduction des dimensions au niveau des règles de dessin (progrès dans la photolithogravure, dans les procédés de fabrication) soit le facteur principal de progrès dans la densité d'intégration des mémoires, les astuces de circuiterie peuvent aussi donner des résultats significatifs. Le principe de stockage de 2 bits par cellule en est un exemple. Avec les technologies MOS actuelles, utilisant un niveau métallique, le pas de métallisation fixe le facteur de répétition des cellules de la ROM. Dans ce pas, il est possible de placer les transistors de taille différente.

Le principe des ROM 4-valuées peut être résumé ainsi :

Le transistor mémoire peut avoir 1 parmi 4 niveaux d'impédance tel que

$$
Z_{i} \in\left\{Z_{0}, Z_{1}, Z_{2}, Z_{3}\right\}
$$

et $Z_{i+1}>Z_{i}$.
Trois transistors de référence ont des impédances $r_{i}$ telles que

$$
r_{i} \in\left\{r_{0}, r_{1}, r_{2}\right\}
$$

et $Z_{i}<r_{i}<Z_{i+1}$ pour $i=0,1,2$.

L'impédance du transistor $T$ est déterminée par comparaison avec l'impédance des trois transistors de référence (Fig. 1).

Il y a une seule comparaison telle que

$$
r_{i}<Z<r_{i+1} \text {. }
$$

Le tableau I donne la relation entre le transistor mémoire, les sorties $X_{0}, X_{1}, X_{2}$ des trois comparateurs et les sorties binaires $d_{0}$ et $d_{1}$.

Dans cet article, nous montrons que les comparateurs peuvent être conçus avec la technologie utilisée pour le projet CMP français (technologie NMOS L3 du CNET-Meylan), avec les règles de dessin simplifiées en $\lambda$ du CMP français.

Les notations sont les suivantes :

$L_{\mathrm{d}}, W_{\mathrm{d}}$ : longueur et largeur dessinées

$L_{\mathrm{e}}, W_{\mathrm{e}}$ : longueur et largeur électriques

$L_{\mathrm{D}} \quad$ : diffusion latérale

$S \quad$ : facteur de shrink (réduction d'échelle).

Les relations suivantes sont utilisées :

$$
\begin{aligned}
& L_{\mathrm{e}}=L_{\mathrm{d}} \times S-2 L_{\mathrm{D}} \pm \Delta L_{\mathrm{e}} \\
& W_{\mathrm{e}}=W_{\mathrm{d}} \times S \pm \Delta W_{\mathrm{e}}
\end{aligned}
$$




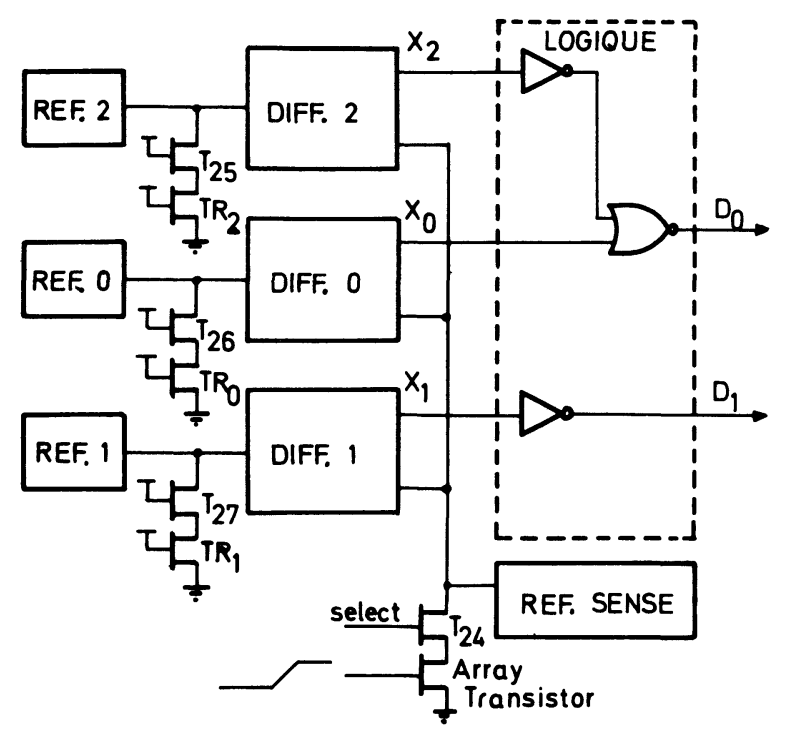

Fig. 1. - Principe de lecture d'une cellule-mémoire : le transistor T24 sélectionne un point-mémoire (noté array transistor).

[Basic scheme for reading a memory cell (the $\mathrm{T}_{24}$ transistor selects a memory transistor referred to as array transistor).]

Tableau I. - Codage des 4 états d'une cellule-mémoire multivaluée.

[Coding of the 4 values of a multivalued memory cell.]

\begin{tabular}{|c|c|ccc|cc|}
\hline Transistor & $\begin{array}{c}\text { Largeur } \\
\text { du transistor }\end{array}$ & $X_{0}, X_{1}, X_{2}$ & $d_{1}, d_{0}$ \\
\hline $\mathrm{T}_{1}$ & $W_{1}$ & 1 & 1 & 1 & 0 & 0 \\
$\mathrm{~T}_{2}$ & $W_{2}$ & 0 & 1 & 1 & 0 & 1 \\
$\mathrm{~T}_{3}$ & $W_{3}$ & 0 & 0 & 1 & 1 & 1 \\
$\mathrm{~T}_{4}$ & $W_{4}$ & 0 & 0 & 0 & 1 & 0 \\
\hline
\end{tabular}

avec $S=0,9$

$$
\| \begin{aligned}
& \Delta L_{\mathrm{e}}=0,1 \mu \mathrm{m} \\
& \Delta W_{\mathrm{e}}=0,2 \mu \mathrm{m} \\
& L_{\mathrm{D}}=0,4 \mu \mathrm{m} .
\end{aligned} .
$$

\section{Conception des comparateurs.}

Selon les règles de dessin simplifiées [5], dites règles de Mead et Conway, la dimension de la cellule ROM binaire est $7 \lambda \times 7 \lambda$, comme le montre la figure 2 . La largeur minimum d'un transistor est $2 \lambda$.

La largeur maximum est $5 \lambda$, différence entre $7 \lambda$ (pas) et $2 \lambda$ (distance minimale entre 2 diffusions). Dans une ROM binaire, les transistors ont tous la même largeur. La figure 2 montre l'exemple 4-valué. Dans le projet CMP, $\lambda=2,5 \mu \mathrm{m}$.

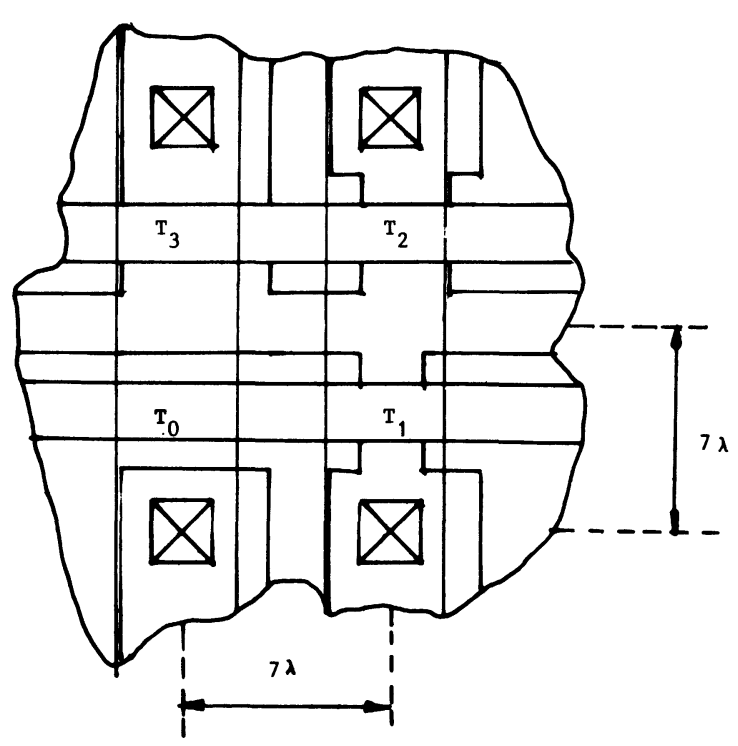

Fig. 2. - Dessin de 4 points mémoire voisins présentant les 4 tailles possibles de transistor.

[Lay out of 4 memory cells showing the 4 transistor sizes.]

Le schéma du comparateur est donné figure 3. Il se décompose en plusieurs blocs fonctionnels :

- les circuits de conversion impédance-tension $\left(T_{17}\right.$ à $T_{19}, T_{21}$ à $\left.T_{23}\right)$. Ils délivrent les tensions $V_{R}$ correspondant à l'impédance du transistor de référence et $V_{\mathrm{S}}$ correspondant à l'impédance du transistor du plan mémoire;

- un amplificateur différentiel $\left(T_{5}\right.$ à $\left.T_{9}\right)$;

- deux circuits suiveurs $\left(\mathrm{T}_{10}-\mathrm{T}_{11} ; \mathrm{T}_{12}-\mathrm{T}_{13}\right)$;

- un amplificateur de sortie $\left(\mathrm{T}_{14}-\mathrm{T}_{15}\right)$.

Il délivre une tension de sortie $X_{\text {out }}$ en fonction des tensions de sortie décalées de l'amplificateur différentiel.

L'étude de l'influence de la taille des différents transistors de chaque bloc a été étudiée avec des programmes écrits en Basic sur ordinateur de poche Casio PB-100, puis avec des programmes écrits en langage $\mathrm{C}$ sur Mini-6 Bull utilisant les mêmes algorithmes.

Nous avons utilisé les équations du modèle 1 de Spice $2 \mathrm{G}$ (équations de Schichman et Hodges), avec les paramètres de simulation donnés sur le tableau II. Les paramètres technologiques sont ceux fournis pour le projet CMP, avec notamment les valeurs suivantes des tensions de seuil des transistors

$$
\begin{aligned}
0,4 \mathrm{~V} & <V_{\mathrm{Te}}<0,9 \mathrm{~V} \\
-2,1 \mathrm{~V} & <V_{\mathrm{Td}}<-1 \mathrm{~V} .
\end{aligned}
$$

Avec ce choix, les dimensions des transistors du plan mémoire et des transistors de référence sont données sur le tableau III. Les caractéristiques détaillées des différents blocs des comparateurs, qui expliquent les dimensionnements choisis sont donnés en [6]. Le tableau IV donne les sorties des compa- 


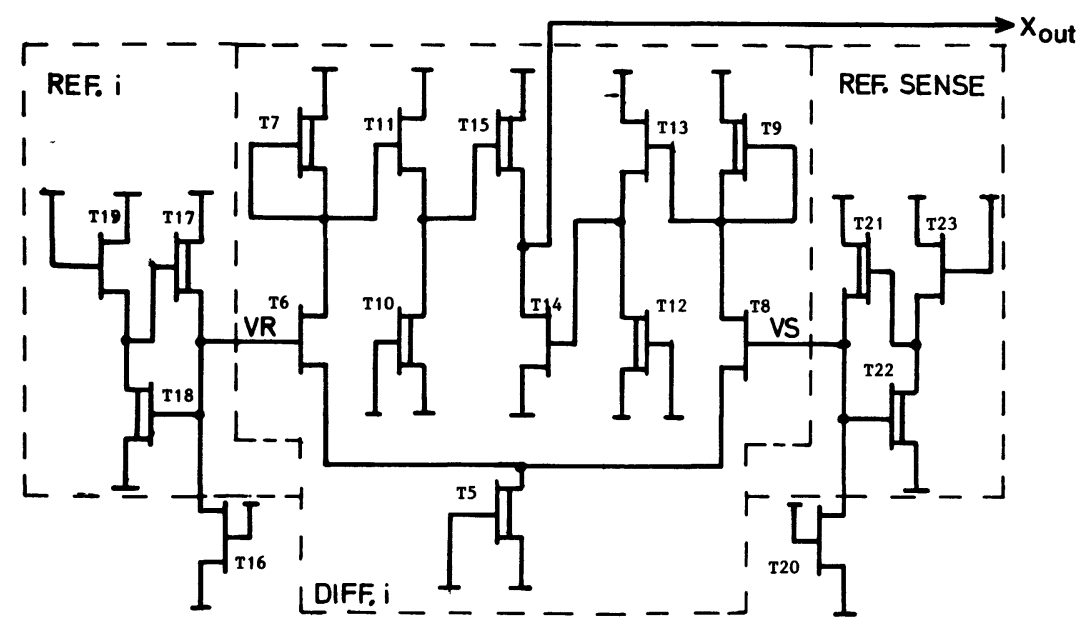

Fig. 3. - Schéma électrique d'un comparateur. $T_{20}$ est le transistor mémoire sélectionné. $T_{16}$ est l'un des transistors de référence.

[Comparator scheme. $T_{20}$ is the selected array transistor. $T_{16}$ is one of the reference transistor.]

Tableau II. - Paramètres électriques du CMP-NMOS.

[Electrical parameters of the French NMOS-MPC.]

- model te nmos level $=1 \quad$ vto $=0.6 \quad \mathrm{kp}=4.4 \mathrm{e}-5 \quad$ gamma $=0.32 \quad \mathrm{phi}=0.57 \quad$ lambda $=0.1$

$+\mathrm{pb}=0.7 \quad \operatorname{cgso}=2.75 \quad \mathrm{e}-10 \quad \operatorname{cgdo}=2.75 \quad \mathrm{e}-10 \quad \operatorname{cgbo}=4.8 \quad \mathrm{e}-10 \quad \mathrm{rsh}=30 \quad \mathrm{cj}=1 \quad \mathrm{e}-4$ is=1 e -14

$+1 \mathrm{~d}=0.4 \mathrm{u}$

- model td nmos level $1=1 \quad$ vto $=-1 \quad \mathrm{kp}=4.4 \mathrm{e}-5 \quad$ gamma $=0.32 \quad \mathrm{phi}=0.57 \quad$ lambda $=0.1$

$+\mathrm{pb}=0.7 \quad \operatorname{cgso}=2.75 \quad \mathrm{e}-10 \quad \operatorname{cgdo}=2.75 \quad \mathrm{e}-10 \quad \operatorname{cgbo}=4.8 \quad \mathrm{e}-10 \quad \mathrm{rsh}=30 \quad \mathrm{cj}=1 \quad \mathrm{e}-4 \quad$ is $=1 \quad \mathrm{e}-14$

$+\mathrm{ld}=0.4 \mathrm{u}$

Tableau III. - Dimensionnement des transistors du comparateur.

[Transistor sizes of the comparator.]

\begin{tabular}{|l|rl|}
\hline Transistors & $\cdot W_{\mathrm{d}}$ & $L_{\mathrm{d}}$ \\
\hline $\mathrm{T}_{1}\left(\mathrm{~T}_{20}\right)$ & 0 & 2 \\
$\mathrm{~T}_{2}{ }^{\prime \prime}$ & 2 & 2 \\
$\mathrm{~T}_{3}{ }^{\prime \prime}$ & 3 & 2 \\
$\mathrm{~T}_{4}^{\prime \prime}$ & 5 & 2 \\
$\mathrm{~T}_{\mathrm{R} 1}\left(\mathrm{~T}_{16}\right)$ & 2 & 3 \\
$\mathrm{~T}_{\mathrm{R} 2}^{\prime \prime}$ & 4 & 3 \\
$\mathrm{~T}_{\mathrm{R} 3}^{\prime \prime}$ & 4 & 2 \\
$\mathrm{~T}_{17-21}$ & 10 & 2 \\
$\mathrm{~T}_{19-23}$ & 10 & 2 \\
$\mathrm{~T}_{18-22}$ & 2 & 2 \\
$\mathrm{~T}_{7-9}$ & 2 & 2 \\
$\mathrm{~T}_{6-8}$ & 40 & 2 \\
$\mathrm{~T}_{5}$ & 2 & 2 \\
$\mathrm{~T}_{11-13}$ & 4 & 2 \\
$\mathrm{~T}_{10-12}$ & 2 & 2 \\
$\mathrm{~T}_{15}$ & 2 & 2 \\
$\mathrm{~T}_{14}$ & 10 & 2 \\
\hline
\end{tabular}

rateurs pour les variations extrêmes des tensions de seuil des transistors.

Des simulations dynamiques correspondant au circuit figure 1 ont été réalisées avec Spice 2 G (en utilisant le modèle 1 avec les paramètres dans le tableau II). Elles donnent le temps de retard entre l'entrée du transistor de sélection et les sorties $d_{0}-d_{1}$, avec les conditions de simulation suivantes :

- les inverseurs et les circuits ont un rapport de géométrie de 4 . La charge capacitive des sorties $d_{0}$ et $d_{1}$ est $0,2 \mathrm{pF}$;

- le temps de montée sur le transistor de sélection est 80 ns. Elle correspond à la pente du signal à l'extrémité d'une longue ligne de polysilicium.

Sous ces hypothèses, le temps de retard est de 42 ns.

Le tableau $\mathrm{V}$ donne les temps de retard pire cas pour différentes valeurs de $V_{\mathrm{Td}}$ et $V_{\mathrm{Te}}$. Ce tableau montre que le temps de retard dépend principalement de la tension de seuil du transistor déplété.

\section{Implantation CMP.}

La ROM 4-valuée a été implantée selon l'organisation $1 \mathrm{~K} \times 8$ bits. Pour évaluer la surface de sili- 
Tableau IV. - Niveaux électriques de sortie des comparateurs pour différentes tensions du seuil des transistors enrichis et déplétés.

[Comparator outputs levels for different threshold levels of enhancement mode and depletion mode transistors.]

\begin{tabular}{|c|c|c|c|c|c|c|c|c|c|}
\hline$T_{R}$ & $V_{\mathrm{s}}$ & $V_{\mathrm{R} 0}$ & $V_{\mathrm{R} 1}$ & $V_{\mathrm{R} 2}$ & $X_{0}$ & $X_{1}$ & $X_{2}$ & $d_{1}$ & $d_{0}$ \\
\hline $\mathrm{T}_{0}$ & 3,32 & 2,28 & 1,97 & 1,74 & 3,82 & 3,94 & 3,96 & 0,11 & 0,11 \\
\hline $\mathrm{T}_{1}$ & 2,07 & $"$ & $"$ & $"$ & 0,16 & 2,84 & 3,96 & 0,16 & 5 \\
\hline $\mathrm{T}_{2}$ & 1,88 & $"$ & $"$ & $"$ & 0,13 & 0,36 & 3,19 & 5 & 5 \\
\hline $\mathrm{T}_{3}$ & 1,62 & " & " & $"$ & 0,13 & 0,15 & 0,37 & 5 & 0,09 \\
\hline \multicolumn{10}{|c|}{$V_{\mathrm{Te}}=0,6$} \\
\hline
\end{tabular}

\begin{tabular}{|l|c|ccc|ccc|c|c|}
\hline $\mathrm{T}_{\mathrm{R}}$ & $V_{\mathbf{S}}$ & $V_{\mathrm{R} 0}$ & $V_{\mathrm{R} 1}$ & $V_{\mathrm{R} 2}$ & $X_{0}$ & $X_{1}$ & $X_{2}$ & $d_{1}$ & $d_{0}$ \\
\hline $\mathrm{T}_{0}$ & 3,65 & 2,55 & 2,21 & 1,95 & 3,99 & 3,99 & 3,99 & 0,26 & 0,26 \\
$\mathrm{~T}_{1}$ & 2,32 & $\prime \prime$ & $\prime \prime$ & $\prime \prime$ & 0,82 & 2,31 & 3,31 & 0,61 & 5 \\
$\mathrm{~T}_{2}$ & 2,11 & $\prime \prime$ & $\prime \prime$ & $\prime \prime$ & 0,59 & 1,24 & 2,38 & 4,77 & 5 \\
$\mathrm{~T}_{3}$ & 1,82 & $\prime$ & $\prime$ & & 0,59 & 0,81 & 1,14 & 5 & 0,21 \\
\hline \multicolumn{10}{c|}{$V_{\mathrm{Te}}=0,9$} \\
\hline
\end{tabular}

\begin{tabular}{|c|c|c|c|c|c|c|c|c|c|}
\hline$T_{R}$ & $V_{\mathrm{S}}$ & $V_{\mathrm{R} 0}$ & $V_{\mathrm{R} 1}$ & $V_{\mathrm{R} 2}$ & $X_{0}$ & $X_{1}$ & $X_{2}$ & $d_{1}$ & $d_{0}$ \\
\hline $\mathrm{T}_{0}$ & 2,31 & 2,00 & 1,73 & 1,53 & 3,52 & 3,59 & 3,59 & 0,07 & 0,07 \\
\hline $\mathrm{T}_{1}$ & 1,82 & $"$ & $"$ & $"$ & 0,07 & 3,34 & 3,59 & 0,08 & 5 \\
\hline $\mathrm{T}_{2}$ & 1,66 & " & " & " & 0,06 & 0,18 & 3,52 & 5 & 5 \\
\hline $\mathrm{T}_{3}$ & 1,43 & $"$ & " & " & 0,06 & 0,09 & 0,20 & 5 & 0,05 \\
\hline \multicolumn{10}{|c|}{$V_{\mathrm{Te}}=0,9$} \\
\hline
\end{tabular}

\begin{tabular}{|c|c|c|c|c|c|c|c|c|c|}
\hline$T_{R}$ & $V_{\mathrm{S}}$ & $V_{\mathrm{R} 0}$ & $V_{\mathrm{R} 1}$ & $V_{\mathrm{R} 2}$ & $X_{0}$ & $X_{1}$ & $X_{2}$ & $d_{1}$ & $d_{0}$ \\
\hline $\mathrm{T}_{0}$ & 3,91 & 2,69 & 2,32 & 2,05 & 3,78 & 4,24 & 4,37 & 0,21 & 0,24 \\
\hline $\mathrm{T}_{1}$ & 2,45 & $"$ & $"$ & "I & 0,46 & 1,61 & 3,88 & 0,79 & 4,99 \\
\hline $\mathrm{T}_{2}$ & 2,22 & " & " & " & 0,33 & 0,77 & 1,80 & 4,74 & 4,90 \\
\hline $\mathrm{T}_{3}$ & 1,91 & " & " & " & 0,32 & 0,38 & 0,75 & 5 & 0,19 \\
\hline
\end{tabular}

\begin{tabular}{|c|c|c|c|c|c|c|c|c|c|}
\hline $\mathrm{T}_{\mathrm{R}}$ & $V_{\mathrm{S}}$ & $V_{\mathbf{R} 0}$ & $V_{\mathrm{R} 1}$ & $V_{\mathrm{R} 2}$ & $X_{0}$ & $X_{1}$ & $X_{2}$ & $d_{1}$ & $d_{0}$ \\
\hline $\mathrm{T}_{0}$ & 3,17 & 2,17 & 1,88 & 1,67 & 3,24 & 3,77 & 3,87 & 0,06 & 0,07 \\
\hline $\mathrm{T}_{1}$ & 1,98 & $"$ & $"$ & $"$ & 0,98 & 3,30 & 3,85 & 0,07 & 5 \\
\hline$T_{2}$ & 1,80 & " & " & " & 0,08 & 0,13 & 3,81 & 5 & 5 \\
\hline $\mathrm{T}_{3}$ & 1,56 & " & " & " & 0,07 & 0,07 & 0,10 & 5 & 0,04 \\
\hline
\end{tabular}

cium et les performances dynamiques, nous avons également implanté une ROM binaire $1 \mathrm{~K} \times 8$ bits.

La ROM binaire a 64 lignes et 16 colonnes. Les blocs fonctionnels sont : le décodeur de lignes, les circuits de puissance pour l'attaque des lignes en poly, le plan mémoire, le décodeur de colonne et la logique de contrôle des plots de sortie (chip select). La ROM 4-valuée a $4 \times 1 \mathrm{~K}$ cellules, avec 64 lignes 
Tableau V. - Temps d'accès simulés, évalués pour différentes températures et tension du seuil.

[Simulated access times, evaluated for different temperatures and threshold levels.]

\begin{tabular}{|l|l|c|c|}
\hline$V_{\mathrm{Te}}$ & $V_{\mathrm{Td}}$ & $T=27^{\circ} \mathrm{C}$ & $T=70^{\circ} \mathrm{C}$ \\
\hline 0,9 & $-2,1$ & $45 \mathrm{~ns}$ & $43 \mathrm{~ns}$ \\
0,9 & -1 & $55 \mathrm{~ns}$ & $55 \mathrm{~ns}$ \\
0,6 & $-2,1$ & $38 \mathrm{~ns}$ & $38 \mathrm{~ns}$ \\
0,6 & $-1,8$ & $37 \mathrm{~ns}$ & $40 \mathrm{~ns}$ \\
0,6 & $-1,4$ & $42 \mathrm{~ns}$ & $43 \mathrm{~ns}$ \\
0,6 & $-1,2$ & $42 \mathrm{~ns}$ & $45 \mathrm{~ns}$ \\
0,6 & -1 & $45 \mathrm{~ns}$ & $50 \mathrm{~ns}$ \\
\hline
\end{tabular}

et 16 colonnes. Les blocs fonctionnels sont les mêmes que ceux de la ROM binaire, avec en plus 4 fois 3 comparateurs.

3.1 Caractéristiques dynamiques de la ROM BINAIRE. - Les caractéristiques dynamiques de la ROM binaire ont été évaluées en considérant l'effet des différents blocs montrés figure 4. L'effet des longues lignes de polysilicium a été modélisé avec des circuits RC. L'effet résistif des masses réalisées en diffusion a été pris en compte.

Deux simulations ont été réalisées, pour le cas nominal $\left(V_{\mathrm{Te}}=0,6 \mathrm{~V} ; V_{\mathrm{Td}}=-1,4 \mathrm{~V}\right.$ et $\left.27^{\circ} \mathrm{C}\right)$ et pour le pire cas de vitesse $\left(V_{\mathrm{Te}}=0,9 \mathrm{~V} ; V_{\mathrm{Td}}=-1 \mathrm{~V}\right.$ et $70^{\circ} \mathrm{C}$ ).

Pour le cas nominal, le temps de retard mesuré au niveau 1,25 $\mathrm{V}$ entre l'entrée de l'adresse et la sortie avec une charge de $10 \mathrm{pF}$ est de $70 \mathrm{~ns}$. Le temps de

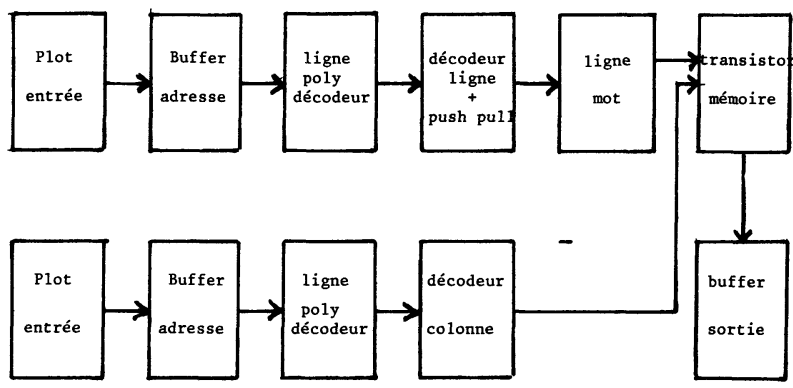

Fig. 4. - Schéma synoptique de la mémoire permettant d'évaluer les temps de propagation dans le circuit.

[Simulation scheme to evaluate ROM access time.] retard dans la longue ligne de poly est environ $15 \mathrm{~ns}$. (Dans le cas 4-valuée, il est moindre avec $4 \times 1 \mathrm{~K}$ cellules au lieu de 8.) La pente du signal à l'entrée du transistor de sélection est de $16 \mathrm{~ns} / \mathrm{V}$. Les valeurs pire cas sont $88 \mathrm{~ns}$ (adresse entrée-sortie) et $16 \mathrm{~ns} / \mathrm{V}$ (signal d'entrée du transistor de sélection).

Ces valeurs donnent un ordre de grandeur des performances dynamiques, car l'utilisation du modèle 1 de Spice (canaux longs) n'est plus valable avec les longueurs de canaux utilisés $\left(L_{\mathrm{e}}=3,7 \mu \mathrm{m}\right.$ pour $L_{\mathrm{d}}=2 \lambda$ ).

3. 2 CaRACtéRISTIQUeS DYNAMIQUeS DE LA ROM 4VALUÉE. - Les performances des comparateurs ont été présentés dans le paragraphe précédent.

Pour une ROM binaire $4 \times 1 \mathrm{~K}$ cellule, le temps de propagation entrée-sortie est $36 \mathrm{~ns}$ et la pente du signal d'entrée du transistor de sélection est $5 \mathrm{~ns} / \mathrm{V}$.

3. 3 Comparaison. - Pour le cas nominal, le temps de propagation total dans la ROM 4-valuée serait inférieur à $78 \mathrm{~ns}$ ( $36 \mathrm{~ns}+42 \mathrm{~ns})$. En effet, le comparateur a été stimulé avec l'hypothèse d'un signal d'entrée de $16 \mathrm{~ns} / \mathrm{V}$ au lieu de $5 \mathrm{~ns} / \mathrm{V}$. La valeur $42 \mathrm{~ns}$ est donc pessimiste. L'augmentation du temps de propagation est d'environ $12 \%$ (78 ns au lieu de 70).

La taille de la ROM binaire est d'environ $1425 \lambda \times$ $965 \lambda$ soit $1,375 \times 10^{6} \lambda^{2}$.

La taille de la ROM 4-valuée est d'environ $1025 \lambda \times$ $1000 \lambda$ soit $1,025 \times 10^{6} \lambda^{2}$.

Le gain de surface est d'environ $25 \%$.

\section{Conclusion.}

Nous avons montré qu'une ROM 4-valuée peut être conçue avec la filière CMP (Circuit Multi-Projets) en utilisant des règles de dessin en $\lambda$, et en tenant compte des dispersions possibles (tensions de seuil des transistors, dispersions sur les longueurs et largeurs électriques des transistors) avec la technologie utilisée pour le CMP. Nous avons poursuivi ce travail en vérifiant que les comparateurs pouvant être conçus pour d'autres valeurs de $V_{\text {Td }}$, et en utilisant des équations de courant tenant compte des effets de petites géométries des transistors. Cette étude, qui n'a pas été présentée dans cet article, valide la faisabilité d'une ROM 4-valuée avec des règles de conception en $\lambda$. La ROM 4-valuée et la ROM binaire sont en cours de fabrication.

\section{Bibliographie}

[1] Stark, M., Two bits per cell ROM, IEEE Compcon Spg 81. Proceedings, Feb. 81, p. 209-212.

[2] Donoghue, B., Bormann, Ai. and Cosentino, S., Variable geometry packs 2 bits into every ROM cell. Electronics 24 (1983) 121-123.

[3] SiLıo, C. B., Control store applications for multivalued circuits, IEEE Compcon Fall 82. Proceedings, Sept. 82, p. 148-150.

[4] Vladimirescu, A. and LiU, S., The simulation of MOS integrated circuits using SPICE 2, ERL Memo $\mathrm{n}^{\circ}$ ERL M 80/7. University of California Berkeley, Feb. 80 .

[5] Mead, C. and Conway, L., Introduction to VLSI systems (Adison \& Wesley).

[6] Lavelle, E. and Etiemble, D., Improved Sense Amplifiers For 4-valued ROMS, Proc. 1984 International Symposium on Multiple Valued Logic Winnipeg, Canada, May 84. Rapport Recherche Equipe Méthodologie et Architecture des Systèmes Informatiques, Paris $6 \mathrm{e}$. 